\title{
AMPUTATIONS OF THE EXTREMITIES IN CASES OF WAR WOUNDS.
}

\author{
By SEYMOUR BARLING, C.M.G., F.R.C.S. \\ (Professor of Surgery in the University of Birmingham.)
}

\begin{abstract}
Although the operation of amputation of a limb calls for no great surgical skill, the decision that ablation is necessary in a given case is a serious one requiring good surgical judgment and experience to weigh up the relative merits of conservative treatment as opposed to amputation; the prospect of successful conservation, and the functional value of the limb ultimately if conservation should be successful. If mature judgment and experience are lacking, this is one of those problems in which consultation with a colleague may be very helpful. This is perhaps especially so when secondary amputation is being considered in a case which we have been watching for some time, which is perhaps tending to lose ground and one in which our attention to details has warped our judgment on its broader aspects:
\end{abstract}

Two factors have combined in the last twenty-five years to modify considerably our traditional attitude towards a procedure which is probably as old as any in surgery, and as a result the problem has been simplified. The numbers of amputations resulting from the last war and the problems arising from their after care was a clinical experiment on a vast scale, and the results of research and investigation carried out under the Ministry of Pensions at Roehampton and at the various limb-fitting centres are now available for our guidance. ${ }^{(1)}$ Furthermore, modifications in the design and type of artificial limbs arose out of the same experiment. As a result of this opportunity of studying large groups of different types of amputation, carried out for the most part on healthy adults, we are able to assess the value of each type as revealed in its functional efficiency and in its capacity to stand up to daily wear and tear over a long period. The coming of the metal artificial limb and the research undertaken into the mechanical problems involved, gave facilities to the limb maker which allowed improvements in design not previously obtainable and these contribute to a better functional result. At the same time the standardising of the metal limbs has to some extent tended to standardise the type of operation which precedes the fitting of the limb. Inasmuch as the surgeon and limb fitter have one end in view, the functional efficiency of the patient, it is not asking too much perhaps that the surgeon should have an understanding of the other partner's difficulties in dealing with the problem. Surgeons have rather tended in the past to carry out their share of the service regardless of the views of the limb maker, merely calling him in later to deal as best he may with what they have produced. A personal knowledge of the mechanical principles involved in the functioning of artificial limbs, and some acquaintance with the point of view of patients who wear them, form an important part of the surgeon's education.

Whilst undoubtedly modern tendencies in manufacture have, to some extent, standardised limbs, yet it must be clearly understood that every patient with an artificial limb is an individual problem. The psychology of the patient, especially in his capacity for adaption, his occupation, no less than the length of the stump, its shape and variations in the site of scars, and its capacity to stand up to daily wear and tear, are all individual problems only to be solved by individual attention. 


\section{The Stump.}

The action of the stump as a lever is its primary function, and as such, length is of the greatest importance, if too short its area of contact with the socket of the limb is too slight and the leverage insufficient to make the proximal joint effective. It may equally fail if too long. Its vitality and capacity to stand up to its work depend on its blood supply and this may be imperilled if it is too long. In the lower limb particularly the piston action of the stump as it is thrust into and slightly withdrawn from the socket with the movements of flexion and extension as each step is taken, demands a high state of nutrition in all tissues of the stump. For the same reason all scars are sources, of weakness, and the scar of the amputation should as far as possible be planned to avoid adhesion to the bone and to be out of the way of direct pressure. Also amputations carried out with too fine a margin of healthy tissue at the site of disease or injury often fail and call for a further amputation after some years of use, the lowered resistance of the damaged tissue eventually giving rise to pain and ulceration.

During the period of healing of the stump and for the first month the parts should be left at rest as far as possible, but care should be taken to prevent the joints becoming stiff or the stump assuming the position of flexion-occasional active or passive movements and attention to posture are sufficient for this. At the end of this first period more active measures should be taken and regular exercises carried out to improve the condition of muscles and joints; shrinkage of the parts sets in and continues for some months and not until this process has become stabilised is the stump fit for its final fitting of an artificial limb. Some time before this, however, in two or three months in favourable cases, it is desirable to fit a temporary limb of simple pylon type with plaster socket to accustom the patient to some of the problems arising from the new position and to promote hardening and shrinkage of the stump. The socket for the stump will need adjustment as the limb shrinks, or the patient may wear several woollen socks to keep the stump fitting snugly in its socket, this being essential for comfort and efficiency.

The ideal to aim at is a stump that tapers somewhat towards its distal extremity, is well covered but without bulky masses of tissue, and has a normal range of movement at its proximal joint. It should not be unduly sensitive to pressure.

Bandaging the stump firmly from the tip upwards with a flannel bandage will assist in moulding it into the required shape and hasten stabilisation. Attention to such details is essential if the stump is to be one that will carry the patient through the rest of his life in comfort and avoid the disability entailed by having to discard the artificial limb from time to time or submit to re-amputation.

\section{Indications for Amputation.}

It is convenient to consider these as they may arise at three different stages following the injury:-

(a) Primary amputation.

(b) Amputation during the stage of progressive sepsis.

(c) Secondary amputation.

(A). Primary amputation. It is difficult to lay down hard and fast rules as to amputation in the first twelve hours after injury. In some cases the bones and soft parts are so badly lacerated and contused that the decision is easy and after 
a short period of resuscitation the limb should be removed as its continued presence increases the danger of prolonged shock which will continue until amputation has been carried out.

The decision to amputate will often have to be taken as the result of findings when the patient is examined under an anæsthetic, but previous to this, much may be learnt from a preliminary examination when the presence of great comminution of bones and damage to joints, the failure of the pulse and circulation in the distal parts of the limb as well as evidence of serious nerve injury, may indicate the necessity for amputation.

The presence of other serious major injuries, the age of the patient, and the absence of satisfactory conditions for after-treatment in the immediate future may, at times, turn the scale in favour of amputation.

High velocity projectiles often inflict injuries at a distance from the apparent tract of the projectile and this is most deceptive to the surgeon accustomed to deal with the ordinary civilian injury such as results from machinery and motor accidents. As a result, secondary projectiles (fragments of bone, etc.) cause widespread damage in the depths of the wound, vessels and nerves are torn, and widespread hæmorrhage spreads along intermuscular planes thus opening channels for sepsis. Furthermore, fragments of clothing or equipment carried deep in the wound implant sepsis in a way totally different from the surface soiling resulting from a motor accident, bad as it may appear.

In the upper limb the surgeon may safely take a more conservative course than in the lower; any portion of fingers or thumb that can be preserved should be safeguarded as being of greater use than any artificial appliance. The lessened risk to life from sepsis and the capacity of the limb to survive severe vascular injury also favour a conservative attitude in the upper limb.

There are two points in the anatomy of the lower limb which must be borne in mind by the surgeon when considering the prospect of successful conservation after injury. The large muscular masses in the thigh when severely injured form a favourable nidus for the development of sepsis, and especially for the anærobic types, this probably arises from the favourable growth pabulum from the released muscle sugars. Furthermore, damage to the long muscle bellies with their blood supply running from end to end may result in long strands of muscle tissue being deprived of their blood supply and thus open to transmit sepsis. The second point is the precarious vascular conditions in the leg and foot resulting from injuries in the neighbourhood of the popliteal space.

These factors and the dangers they entail as well as the greater functional value of the artificial leg should make us less reluctant to sacrifice the lower limb than the upper when faced with the decision.

(B). Amputation during the early period of sepsis. This group consists of cases which first come under the surgeon a day or two after injury in which sepsis is already established and those in which the primary conservative treatment has failed. The patient is not only a wounded man but a sick man and if life is to be saved the decision to amputate must be taken promptly.

(1) Cases with rapidly spreading sepsis often with gas forming anærobes; here progress downhill is terribly rapid and only immediate amputation can save life.

(2) Cases which have never really recovered from the initial shock but remain lethargic with a low blood pressure and with slowly ingravescent sepsis in the wound.

(3) Cases in which the circulation in the distal parts of the limb fails, either frank gangrene appears or the low vitality sets up no barrier to infection. 
(C). Secondary amputation. Amputation may become necessary during the later stages of recovery from a variety of causes. The occurrence of acute arthritis in a large joint, the presence of widespread osteomyelitis and pyæmia, the gradual failure of the patient with widespread sepsis, form some of the indications in this group. The recognition that the limb, when recovery does ultimately take place, will have less functional value than an artificial limb may save many months of vain suffering if ablation is carried out, and long standing cases of limb injury should be reviewed from time to time with this point in mind.

\section{The Operation.}

Primary amputation has often to be carried out in the shocked patient and two or three hours spent in resuscitation which should include a blood transfusion in the ex-sanguinated patient, is desirable. At the same time the presence of the damaged limb is a potent source of shock and if the systolic blood pressure is IOO $\mathrm{mm}$. $\mathrm{Hg}$. or over there should not be too much delay before removing it. Gas and oxygen is a satisfactory anæsthetic in such cases. The use of a tourniquet at the root of the limb is convenient and lessens blood loss. In amputations at or near the hip joint, the femoral vessels should be ligated through a vertical incision which afterwards falls into that of the amputation. The exact siting of the flaps must depend on the circumstances present immediately below the amputation. Flaps should, however, be of approximately equal lengths-very long flaps have poor blood supply at their extremity, and the flaps should be so sited that the scar is out of the way of pressure or adhesion to bone.

In the presence of sepsis it is often an advantage to sacrifice an inch or two of stump for the sake of avoiding the complications resulting from the pressure of the limb on such damaged tissues. The guillotine operation is unsatisfactory even as a temporary measure, giving a painful and prolonged convalescence. If sepsis is unavoidable in the stump the flaps should be sutured lightly over paraffin gauze, and in the ordinary case drainage for forty-eight hours is advisable. The main nerve trunks should be drawn down slightly and divided with a knife and allowed to retract. If, as in the sciatic, a considerable vessel is present a light ligature may be tied around the whole nerve but I am not in favour of crushing or ligaturing nerves as a rule. The avoidance of painful movements and excessive stimuli to the stump during the first weeks of convalescence should be aimed at if hyperæsthesia and pain are to be avoided. If long standing sepsis has preceded amputation, a still longer period of rest for the stump should be enforced.

\section{Amputations above the Knee.}

If amputation above the knee is necessary and circumstances permit it, the ideal stump should have a length of ro inches from the tip of the great trochanter, or I2 inches if the patient is tall; this provides a lever of sufficient length and permits of the retention of sufficient muscular tissue to give good abduction and adduction as well as flexion and extension which depend on the ilio-psoas. The total length of the flaps should be slightly greater than $\mathrm{I} \frac{1}{2}$ times the diameter of the limb at section point and they are best of anterior and posterior type and of approximately equal lengths. A circular amputation is satisfactory if preferred, the scar being transverse when sutured. Retraction being greater on the posterior surface of the thigh, the resulting scar will lie somewhat drawn up on the posterior surface of the bone. Under normal circumstances such a stump will be fitted to a socket that takes its bearing from the tissues at the root of the thigh and the ischial tuberosity and permits the fitting of a most efficient prosthesis 
giving very good function. If the stump is longer it has the inherent disadvantages which have been mentioned. If circumstances necessitate a shorter stump every inch should be conserved, for even a length of only 6 inches is, at times, sufficient to permit of a stump controlled limb. If less than a 6 inch stump is available a heavier and less efficient limb is necessary and the stump is no longer of functional value as a lever. In amputations through the hip joint the upper socket for the limb is moulded closely round the corresponding half of the pelvis and must, in order to be efficient, make close contact with it. If amputation is through the joint itself the concavity thus left after removal of the head of the bone renders this fitting very difficult owing to lack of a point $d^{\prime} a p p u i$ for the socket, whereas if the head or neck is left this results in a convexity beneath the scar which gives the instrument maker a point from which to get purchase to steady the socket and thus conduce to good function. If it is possible therefore to conserve the upper part of the femur but not sufficient is available to leave 6 inches (measured from the tip of the great trochanter) it is best to divide the bone at the level of the lesser trochanter. If section is necessarily made higher than this, the portion of bone remaining is still of value even if the section is immediately below the head.

\section{Below the Knee Amputation.}

For the most satisfactory function, free movement of the knee joint is essential and sufficient length of stump to form an efficient lever. Measuring from the top of the tibia 6 inches of bone is desirable, and removal of the limb at this level is the site of election. Shorter stumps of 4 inches are still useful and even a length of less than 2 inches may at times be utilised by the instrument maker as an efficient lever. The scar should be transverse just behind the bone ends, and either antero-posterior flaps or a circular amputation may be used, cut to allow a slightly longer anterior flap. The lower end of the fibula should be divided I inch above the level of the tibia. If circumstances are favourable and the stump free from scars over the tuberosities of the tibia the bearing for the limb may be taken from this area of the bone; failing this, but giving a less efficient prothesis, bearing may be taken from the thigh or from the ischium. If amputation is necessary in the presence of an ankylosed knee joint in whatever position, it is probably best to leave a stump of not more than 4 inches.

\section{Symes Amputation.}

The long stump in below the knee amputations has the inherent disadvantages arising from deficient circulation and does not stand up to wear and tear over long periods, and experience in the last war showed that the Syme amputation had the same disadvantage and in.most of the pensioners re-amputation was required. In some, but not in all cases, failure arose from amputation through septic tissues, where a higher level should have been chosen. In many others, however, this was not the cause, the stump failed owing to poor nutrition of the terminal flap with the result that painful callosities occurred and stretching of the scar made weight-bearing impossible. In many cases in civilian life, however, and especially in children such stumps have given long years of service and there is no doubi that its lessened mutilation compared with a higher amputation through the leg gives it a strong sentimental appeal. Max Page ${ }^{(2)}$ has suggested a slight modification whereby the bones are divided I inch above the ankle joint and the heel flap is fitted snugly over the ends of the bones thus giving a type of stump which will wear better and to which it is easier to fit an artificial limb than in the traditional operation. 


\section{Amputations of the Upper Limb.}

The relative inefficiency in the upper limb of the prosthetic apparatus compared with the good function obtainable in the lower limbs after amputation warrants a strong bias towards conservation when amputation is being considered. Any part of the fingers or thumb that can give prehension against the palm or any possibility of opposition to a fragment of the thumb are more valuable than any artificial limb. When the hand, however, has gone we can consider the problem of the limb fitter and give him the best foundation for his work in providing a suitable stump that will give the most useful function. As in the lower limb so in the upper, amputations through joints are to be avoided as they give a stump which is too long for useful service, whilst the club shaped end provides a difficult problem in fitting the socket.

Whilst the stump should be long enough to provide efficient leverage it must also be freely moveable at the proximal joint and every care must be taken during convalescence to maintain function of muscles and joints.

The ideal stump should taper slightly towards its distal extremity and not be too bulky; scars, wherever present, are sources of weakness for although the stresses the stump has to put up with are less than those in the lower limb they are still considerable in the manual labourer. The scar of the amputation itself is best sited to lie transversely across the bone ends where, if primary union occurs, it may move freely over the adjacent bone. Upper limb stumps are particularly prone to be painful and this is a problem which cannot be considered within the limits of this article except to say that long continued sepsis preceding amputation is one of the potent causes of post-operative pain and should be met by a corresponding long period of rest before fitting the limb. At the same time, the early fitting of a limb gives the patient a more hopeful attitude towards his disability and encourages the nutrition and general function of the stump and these two factors must be carefully balanced in considering the question.

\section{Below the Elbow Amputation.}

An amputation $2 \frac{1}{2}$ inches above the tip of the ulna styloid process gives the most useful stump, and any length of bone that can be preserved up to within 4 or even 3 inches below the internal condyle is useful; if this is not possible then amputation had better be above the elbow joint. Reference has been made to the value of preserving any fragment of a useful hand; short of this it may be possible to amputate at or below the metacarpo-phalangeal joint and this may be carried out, for although such a stump is apt to be too sensitive and of doubtful value in the absence of finger or thumb, it is occasionally of value and may, if useless, be re-amputated.

\section{Amputation above the Elbow.}

The ideal level for amputation here is 3 inches above the elbow joint, but any bone that can be left up to 6 or even 5 inches measured from the tip of the acromion process is of value; above this level the stump is fitted as for a shoulder amputation and loses its value as a lever.

\section{Amputation at or about the Shoulder.}

From a functional point of view it is an advantage to preserve a portion of the upper end of the humerus, for although it is useless as a lever, it preserves the contour of the shoulder and forms anchorage for the shoulder cap which is the basis of the limb; the scar should be as far as possible out of the way of pressure and lie vertically below the acromion.

REPERENCES.

(1). Artificial Limbs and their relation to Amputations. H.M. Stationery Office. London. 1939.

(2). C. Max Page. Brit. Med. Journ. II. 1939. P. 77. 